This document is the accepted manuscript version of the following article:

Jougnot, D., Jiménez-Martínez, J., Legendre, R., Le Borgne, T., Méheust, Y., \& Linde, N. (2018). Impact of smal1-scale saline tracer heterogeneity on electrical resistivity monitoring in fully and partially saturated porous media: insights from geoelectrical milli-fluidic experiments. Advances in water Resources, 113, 295-309.

http://doi.org/10.1016/j.advwatres.2018.01.014

This manuscript version is made available under the CC-BY-NC-ND 4.0 1icense http://creativecommons.org/1icenses/by-nc-nd/4.0/

\title{
Impact of small-scale saline tracer heterogeneity on electrical resistivity monitoring in fully and partially saturated porous media: insights from geoelectrical milli-fluidic experiments
}

Damien Jougnot ${ }^{1}$

Sorbonne Universités, UPMC Université Paris 06, CNRS, EPHE, UMR 7619 METIS Paris, France.

Joaquín Jiménez-Martínez

Department Water Resources and Drinking Water, Swiss Federal Institute of Aquatic Science and Technology, EAWAG, Dubendorf, Switzerland.

Department of Civil, Environmental and Geomatic Engineering, ETH Zurich, Zurich, Switzerland.

Raphaël Legendre, Tanguy Le Borgne and Yves Méheust

Géosciences Rennes (UMR CNRS 6118), University of Rennes 1, Rennes, France.

Niklas Linde

Applied and Environmental Geophysics Group, Institute of Earth Sciences, University of Lausanne, Switzerland.

\begin{abstract}
Time-lapse electrical resistivity tomography (ERT) is a geophysical method widely used to remotely monitor the migration of electrically-conductive tracers and contaminant plumes in the subsurface. Interpretations of time-lapse ERT inversion results are generally based on the assumption of a homogeneous solute concentration below the resolution limits of the tomogram depicting inferred electrical conductivity variations. We suggest that ignoring small-scale solute concentration variability (i.e., at the sub-resolution scale) is a major reason for the often-observed apparent loss of solute mass in ERT tracer studies. To demonstrate this, we developed a geoelectrical milli-fluidic setup where the bulk
\end{abstract}

\footnotetext{
${ }^{1}$ Corresponding author: damien.jougnot@upmc.fr
} 
electric conductivity of a 2D analogous porous medium, consisting of cylindrical grains positioned randomly inside a Hele-Shaw cell, is monitored continuously in time while saline tracer tests are performed through the medium under fully and partially saturated conditions. High resolution images of the porous medium are recorded with a camera at regular time intervals, and provide both the spatial distribution of the fluid phases (aqueous solution and air), and the saline solute concentration field (where the solute consists of a mixture of salt and fluorescein, the latter being used as a proxy for the salt concentration). Effective bulk electrical conductivities computed numerically from the measured solute concentration field and the spatial distributions of fluid phases agree well with the measured bulk conductivities. We find that the effective bulk electrical conductivity is highly influenced by the connectivity of high electrical conductivity regions. The spatial distribution of air, saline tracer fingering, and mixing phenomena drive temporal changes in the effective bulk electrical conductivity by creating preferential paths or barriers for electrical current at the pore-scale. The resulting heterogeneities in the solute concentrations lead to strong anisotropy of the effective bulk electrical conductivity, especially for partially saturated conditions. We highlight how these phenomena contribute to the typically large apparent mass loss observed when conducting field-scale time-lapse ERT.

Keywords: Hydrogeophysics, Petrophysics, Millifluidics, Electrical Conductivity, Unsaturated Flow, Tracer test, Transport in Porous Media, Anisotropy

\section{Introduction}

Geophysical methods are increasingly used in subsurface hydrology. Their main advantages lie in their largely non-invasive nature, their sensitivity to properties of interest, and in that they provide images of the subsurface at a 5 comparatively high spatial resolution [e.g. 1, 2, 3, 4, 5]. A particular empha-

6 sis has been given to geophysical methods with responses that depend on the 
7 electrical resistivity (or the electrical conductivity, its inverse) because electrical

8 resistivity is sensitive to sub-surface properties such as: the lithology (porosity,

9 tortuosity, specific surface area), the presence of fluids in the pore space (water saturation and its spatial distribution), and the pore fluid chemistry (ionic concentrations). The links between physical properties (electrical resistivity) and hydrological properties and state variables of interest are described by petrophysical relationships (for literature reviews, see $[4,6]$ among others).

Resistivity methods can be applied to a wide range of scales, from the laboratory (on centimetric samples) to the field (up to several kilometers). Measurements are achieved by driving a known electrical current between an electrode pair while measuring the resulting voltage between another electrode pair. The electrical resistivity structure of the subsurface can be inferred by electrical resistivity tomography (ERT), which is an inversion process that uses measured electrical resistances from multiple current injection and voltage pairs (e.g., Binley and Kemna [7]). If the measurement process is repeated in time, it is possible to perform time-lapse inversion and, thus, to track temporally-varying 23 processes in the subsurface (e.g. [8]). Time-lapse ERT has been widely applied under both saturated (e.g., [9, 10, 11]) and partially saturated conditions (e.g., $[12,13,14,15])$ using electrodes placed on the ground or in boreholes.

Geophysical data have a limited resolving power, which implies that geophysical tomograms are best understood as spatially-filtered representations of subsurface properties (e.g., Menke [16], Friedel [17]). The "filter width" is often 29 referred to as the resolution and it varies in space and time as a function of experimental design, noise, the actual electrical conductivity distribution and choices made when developing or running an inversion algorithm. In ERT studies, the 32 resolution decreases (the filter width increases) when the distance between the electrodes and the target of interest increase. Day-Lewis et al. [18] highlight the inherent resolution limitations of cross-borehole ERT through a careful nu35 merical and theoretical study. The limited resolution of ERT tomograms can (if 36 ignored) lead to important errors when translating inferred resistivity to prop37 erties of interest through petrophysical relationships (e.g., [19, 14, 20, 15]). For 


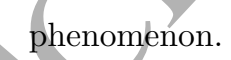

63 The question of how field-scale studies are impacted by sub-resolution flow and transport processes is deeply tied to the physics of these processes. For example, unsaturated flows give rise to gravitational [29] and viscous [30, 31, 32] 66 interface instabilities leading to sub-Darcy-scale fingering. It is now well under67 stood that this fingering is the main reason why Darcy-scale modelling of flows ${ }_{68}$ in the unsaturated zone should consider a dependence of the capillary pressure 
3. the numerical modeling of the electrical problem (section 3); finally, we present

94 and discuss in section 4 the results that we have obtained from tracer tests under fully-saturated and partially-saturated conditions, and how these results can be used to gain insights into how upscaled bulk electrical resistivity is affected by 97 sub-resolution heterogeneity and processes. 
2 system (MegaPlus EP11000, Princeton Instruments) was set to take 1 picture every 2 s to capture the fluid phases and solute dynamics in the flow cell.

The flow cell consists of a single layer of 4500 cylindrical solid grains positioned between two parallel transparent plates separated by a distance equal to the cylinders' height. It is built by soft lithography as follow. Two glass plates 

by weight distilled water/glycerol solution (see [35]). The glycerol increases the viscosity of the solution $\left(\mu_{\mathrm{w}}=3.78 \times 10^{-2} \mathrm{~Pa} \mathrm{~s}\right)$, thereby increasing the 156 viscosity ratio between the wetting and non-wetting phases, and slowing down

are separated by the desired distance using spacers. The space between them is filled with a UV-sensitive polymer (NOA-81). The photomask (resulting from a numerical model of the $2 \mathrm{D}$ compaction of circular grains with diameters distributed according to a Gaussian law of prescribed standard deviation) is then placed on top of the top plate. The mask is transparent where solid grains are to be found, opaque everywhere else. The light coming from the collimated 365 nm UV source passes through the transparent disks in the mask, polymerizing the NOA-81 and giving rise to solid cylindrical grains spanning the vertical gap between the two glass plates. The remnant uncured, still liquid, polymer material is cleaned by flowing through ethanol. The resulting 2-D porous medium is water-wet.

The geometry used herein corresponds to the so-called homogeneous geometry used by Ferrari et al. [44]. The flow cell is closed on two of its lateral sides (facing each other), while the two other lateral sides remain open and constitute the inlet and outlet of the cell. Its length, defined between the inlet and outlet, is $140 \mathrm{~mm}$, its width is $92 \mathrm{~mm}$, and its thickness (equal to the cylinder height) is $0.5 \mathrm{~mm}$ (see [35] for details). The cell is positioned with the glass plates lying horizontal. The vertically-oriented cylindrical grains act as obstacles for the flow of fluids in the cell (Fig. 1a). This 2D geometry has a cross-sectional area of $43.64 \mathrm{~mm}^{2}$ in the direction normal to the average flow direction and typical pore throat and pore sizes of $1.07 \mathrm{~mm}$ and $1.75 \mathrm{~mm}$, respectively. It yields a permeability of $4.32 \times 10^{-9} \mathrm{~m}^{2}$.

The cell is connected to three reservoirs upstream that contain wetting and non-wetting fluids, and to an outlet reservoir downstream. The fluids are injected in the flow cell with syringe pumps at a controlled flow rate. The nonwetting phase is air (Fig. 1a) and the wetting (i.e., liquid) phase is a 60-40\% molecular diffusion. The wetting phase solutions 1 and 2 have different mass concentrations of $\mathrm{NaCl}$ salt $\left(C_{\mathrm{NaCl}}\right)$ and fluorescein $\left(C_{\text {fluo }}\right)$. These solutions are 

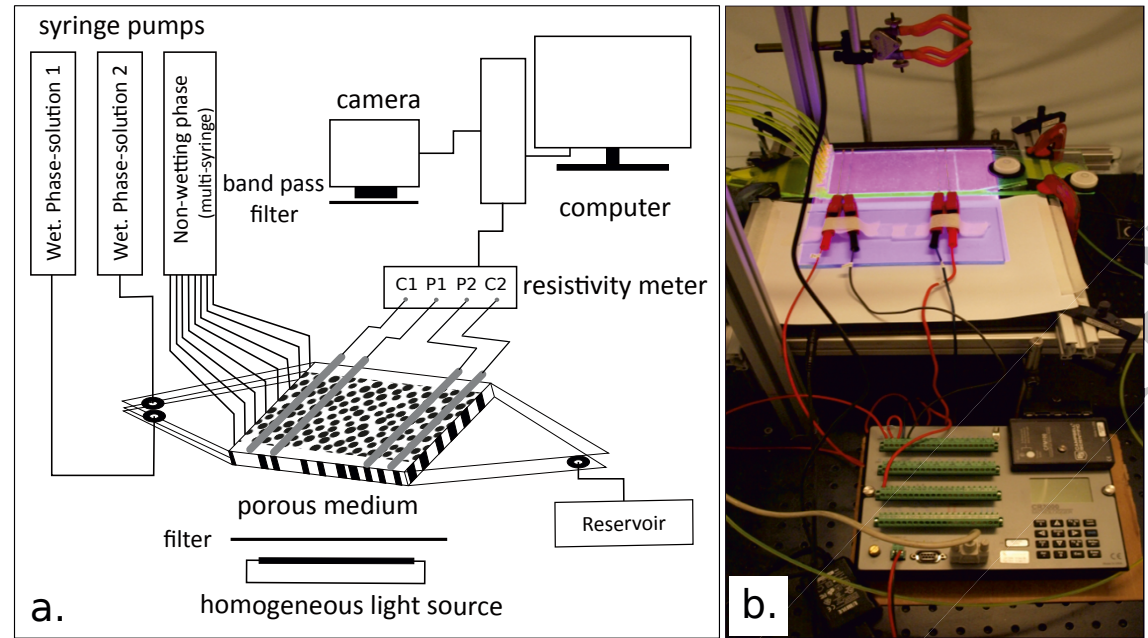

Figure 1: (a) Overall scheme of the setup for the fluorimetric study in the 2D porous medium, featuring the injection systems for air (non-wetting fluid phase), the tracer solution (wetting phase 1), and the background solution (wetting phase 2), as well as the camera and the electrical resistivity monitoring system (modified from [35]). (b) Photography of the geoelectrical milli-fluidic setup.

${ }_{168}$ tivity meter (WTW Cond 340i). Correspondingly, the light intensity of each solution was measured in a cell similar to the one used for the tracer experito ments (i.e., same glass and aperture thickness) but without cylindrical pillars. 11 For the tracer experiments, we chose the background concentration solution $172 \quad\left(C_{\text {fluo }}^{\text {tr }}=5.48 \mathrm{mg} \mathrm{L}^{-1}\right)$ by measuring the light intensity for all the solutions and 

sence of multiple scattering by a factor which is a function of the flow cell's thickness. Hence a discrepancy in the cell thicknesses would lead to exactly this type of effect. Therefore, we have corrected the recorded calibration curve by

selecting the lowest concentration for which the corresponding light intensity was above the detection threshold. Then, we chose the tracer concentration to be $C_{\text {fluo }}^{\operatorname{tr}}=350.8 \mathrm{mg} \mathrm{L}^{-1}$ in to avoid light saturation for the camera. It yields a background and tracer electrical conductivity of $\sigma_{\mathrm{w}}^{\mathrm{bkg}}=0.0055$ and $\sigma_{\mathrm{w}}^{\mathrm{tr}}=0.213 \mathrm{~S} \mathrm{~m}^{-1}$ respectively. We only kept the six solutions with fluorescein mass concentrations in between these two values to establish the calibration curve. Combining these two sets of measurements and using a Piecewise Cubic Hermite Interpolating Polynomial interpolation in between the data, we obtain an empirical curve relating the measured light intensity and the electrical conductivity of the solution $\sigma_{\mathrm{w}}\left(\right.$ in $\left.\mathrm{S} \mathrm{m}^{-1}\right)$ (Fig. 2).

However, when using this calibration curve to infer local solution conductivities inside the flow cell during subsequent tracer tests (which we present in section 4 below), we noticed that the largest conductivity values measured inside the cell were larger than the conductivity of the injected tracer solution. This unphysical result showed that the calibration curve obtained in the flow cell without solid grains (a standard Hele-Shaw cell), was not fully adequate for the tracer experiment cell. We have therefore assumed that a slight difference in the cell thickness, or perhaps the impact of the presence of the translucid solid grains, was responsible for the discrepancy. In particular, given the large fluorescein concentration in the injected tracer, it is not unreasonable to consider that some multiple scattering of the light emitted by the fluorescein may occur in the cell, with a fraction of the emitted light being absorbed by other fluorescein molecules on its way out from the cell, which is possible due to the overlapping emission and absorption spectra of fluorescein (see [45]). Accounting for this multiple scattering yields a prediction of the light intensity transmitted to the camera that is offset from the intensity measured in the abassuming that the light intensity value calibrated in the pure Hele-Shaw cell for a given tracer concentration was offset by a given factor (independent of the 

and P2 is $97 \mathrm{~mm}$ in order to study the largest possible zone of the flow cell, while the C1-P1 and P2-C2 spacings are $8 \mathrm{~mm}$ (Fig. 1). The electrodes consist of a thin layer of copper $(90 \mu \mathrm{m})$. They were inserted 221 222 tal cell. The correction factor of 0.805 has been inferred from the (reasonable) constraint that the maximum conductivity value measured in the cell during the experiment should exactly correspond to the conductivity of the injected tracer solution. The corrected calibration curve is used systematically when inferring local conductivities from light intensities in our experimental cell (Fig.2).

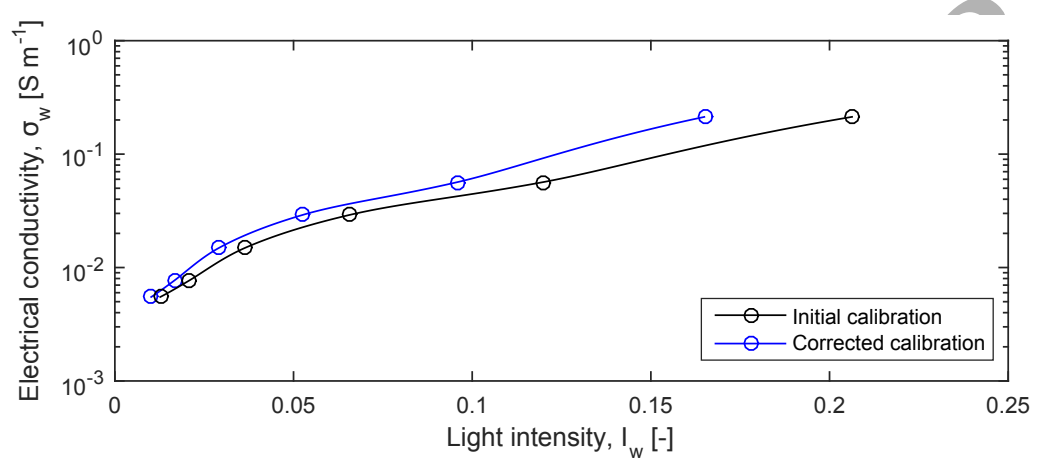

Figure 2: Initial and corrected calibration curves: pore water electrical conductivity $\sigma_{\mathrm{w}}$ as a function of the light intensity $I_{\mathrm{w}}$.

\subsection{Geoelectrical monitoring}

The geoelectric monitoring is performed using a four electrode setup (see Schlumberger [46] for the historical paper, and more recently Binley and Kemna [7] for a more hydrology-oriented introductory text). We inject a current in the two outer electrodes ( $\mathrm{C} 1$ and $\mathrm{C} 2)$ and measure the resulting electrical voltage between the two inner electrodes (P1 and P2) (Fig. 1a). Given that the zone of investigation is localized between $\mathrm{P} 1$ and $\mathrm{P} 2$, we chose not to have equally we at the bottom of the cylinder layer while manufacturing the cell; this ensured good contact with the fluids in the cell without perturbing the flow. We chose

given tracer concentration) with respect to the correct value for the experimen- 
$$
248
$$

${ }^{249}$ to partly fill the medium with air while keeping the liquid phase connected 250 (thereby imposing partially saturated conditions). By varying the injection 251 rates of the fluids, we reached three or more steady state flows with different 252 2 saturations (i.e., proportion of wetting fluid in the porous space). By steady 
state flows we refer to flows for which the spatial distributions of the fluid phases change continuously, but their statistical properties (saturation, distribution of cluster sizes, see [48]) are stationary. The steady state is considered to have been reached when the saturation fluctuates around a plateau value, and the longitudinal and transverse saturation profiles fluctuate around a uniform stationary profile. This can only be measured a posteriori, from the images. After performing measurements with the largest saturation range $\left(S_{\mathrm{w}}\right)$ possible with the setup and experimental protocol (i.e., $S_{\mathrm{w}} \in[0.46 ; 1]$ ), the procedure was repeated with another concentration of the solution (i.e., another $\sigma_{\mathrm{w}}$ ). Note that the lower saturation limit is linked to the connectivity of the liquid phase and its stability overtime; liquid phase connectivity is necessary to allow measurement of the bulk electrical conductivity. During these steps, both the bulk electrical conductivity of the cell and the spatial distribution of the fluid phases were recorded. These first series of measurements provided a set of images and electrical conductivity measurements for different tracer solutions (i.e., different $\left.\sigma_{\mathrm{w}}\right)$ at different saturation degrees.

\subsection{Tracer test procedures}

After these initial experiments, we conducted a tracer test under saturated conditions and three tracer tests under partially saturated conditions.

For the fully saturated test, the medium was first saturated with the background solution $\left(C_{\text {fluo }}^{\mathrm{bkg}}\right.$ and $\left.\sigma_{\mathrm{w}}^{\mathrm{bkg}}\right)$ to obtain a homogeneous initial state. Then, the tracer $\left(C_{\text {fluo }}^{\mathrm{tr}}\right.$ and $\left.\sigma_{\mathrm{w}}^{\mathrm{tr}}\right)$ was injected at a constant rate $\left(1.375 \mathrm{~mm}^{3} \mathrm{~s}^{-1}\right)$. The injection rate was chosen to be low enough to follow the dynamics with the sampling frequency of our acquisition setup. It yields the dimensionless Reynolds and Péclet numbers, $\mathrm{Re}=1.64 \times 10^{-4}$ and $\mathrm{Pe}=241$, respectively. The test was stopped when the measured electrical conductivity reached a constant value (after $\sim 12500 \mathrm{~s}$ ), that is, when an apparent steady-state was reached for the salt concentration field.

For the tracer tests performed under partially-saturated conditions, an unsaturated flow was first imposed by jointly injecting air and the background 
solution at constant flow rates to reach a steady-state flow as explained in section 2.3, with a given liquid saturation of the medium and a given size distribution of air clusters. After stopping the injection of air and background solution, the tracer solution was injected continuously and at a volumetric flow rate that was sufficiently low so that the impact on the previously-established air cluster was minimal $\left(0.277 \mathrm{~mm}^{3} \mathrm{~s}^{-1}\right.$, yielding $\mathrm{Re}=3.79 \times 10^{-4}$ and $\left.\mathrm{Pe}=68\right)$. The experiments were terminated when the measured bulk electrical conductivity of the flow cell reached a constant value (after $\sim 15200 \mathrm{~s}$ for the test presented in the results section).

\section{Modelling approach}

\subsection{From images to effective bulk electrical conductivity}

The experiments described in the previous section provide two kinds of data: images with a light intensity value per pixel, $I(x, y)$, on the one hand, and an effective bulk electrical conductivity of the entire cell, $\sigma^{\text {meas }}$, on the other hand. In this section, we describe how we simulate the effective bulk electrical conductivity from the images in order to compare the computed conductivity, $\sigma^{\text {sim }}$, to the measured one, $\sigma^{\text {meas }}$.

The raw images are first corrected for spatial heterogeneities in the incident light intensity (which is largest at the center of the flow cell). All subsequent data processing is performed on these corrected images. Figure 3 shows the flow chart used to process such a corrected image, and the subsequent electrical field simulations. The flow cell geometry, with the exact geometry of the borders and exact position of each cylinder, is obtained from an image of the medium saturated with a solution at $C_{\text {fluo }}^{\text {bkg }}$ and is stored as a binary image denoted "pore space mask", which defines the pore space: $I_{\text {mask }}=0$ for pixels positioned inside borders and cylindrical grains, and 1 for pixels positioned within the pore space. The electrodes are clearly visible in the images; their positions and geometries are extracted and stored into another mask, the "electrode mask". The porosity of the medium is readily computed from the pore space mask: 


$$
\text { w) }
$$
significant electrical current flow in these materials while allowing convergence of the numerical code. These electrical simulations will help understand ex340 actly where the electrical current is flowing and its links with solute transport

$\phi=0.73$. Subsequent images $(2966 \times 2308$ pixels $)$ acquired during the course of the experiments are used to extract (1) the phase distribution and (2) the map of local conductivities at different times. Image pixels belonging to the air phase are identified as those for which the mask value is $1\left(I_{\text {mask }}=1\right)$ and the recorded light intensity is null $(I=0)$. The liquid (wetting fluid) phase is identified as consisting of pixels for which $I_{\text {mask }}=1$ and $I>0$; we define a light intensity map in the wetting phase, $I_{\mathrm{w}}(x, y)$, equal to 0 outside the water phase, and to $I(x, y)$ inside the water phase. From this image processing, we can monitor the water saturation during the tests by considering the ratio of the number of pixels belonging to the water phase to the number of pixels belonging to the entire pore space. Using the corrected calibration curve discussed in section 2.1 above, we then convert the $I_{\mathrm{w}}(x, y)$ map in to a map of local conductivities.

Using the spatial distributions of the phases and local tracer conductivities, it is possible to simulate the cell's effective electrical bulk conductivity, $\sigma^{\text {sim }}$. This numerical upscaling is based on the pixel distribution of electrical conductivity and is, therefore, limited by the image resolution. Using to the calibration relationship between $\sigma_{\mathrm{w}}$ and the light intensity obtained from the different dilutions as described in paragraph 2.1, each pixel in the image is attributed a given electrical conductivity $\sigma_{\mathrm{w}}(x, y)$ from $I_{\mathrm{w}}(x, y)$. The electrical conductivity of the wetting fluid ranges between $\sigma_{\mathrm{w}}=0.0041$ and $0.2130 \mathrm{~S} \mathrm{~m}^{-1}$. In the simulations, the electrodes were given an electrical conductivity of $\sigma_{\text {elec }}=10^{4}$ $\mathrm{S} \mathrm{m}^{-1}$, which is sufficiently high to obtain practically-constant isopotential values along the electrodes but also small enough to avoid numerical problems. The polymer NOA81 (cylinders and borders) is, similarly to the air phase, a nonconducting material and an arbitrary low electrical conductivity is assigned to both: $\sigma_{\mathrm{NOA} 81}=\sigma_{\text {air }}=10^{-6} \mathrm{~S} \mathrm{~m}^{-1}$, which is a value sufficiently low to avoid processes.

The numerical upscaling of the electrical conductivity consists in solving 
7

$$
\sigma=\frac{S_{\mathrm{w}}^{n}}{F} \sigma_{\mathrm{w}},
$$

the Poisson equation in 2D. We use a modified version of the code MaFlot (www.maflot.com) initially designed to address density-driven Darcy flows [49]. In the simulations, we generate the current flow by imposing an electrical voltage of $1 \mathrm{~V}$ between the current electrode (C1 and C2) (see Fig. 1). The top and bottom boundary conditions are set to electrical insulation. The electrical problem is therefore a boundary problem that can be seen as an analog to determining the effective hydraulic conductivity of a porous medium of heterogeneous permeability field between two reservoirs with a given pressure difference under steady state conditions. Note that imposing a potential difference in the modelling instead of injecting a fixed electrical current presents two main advantages: (1) it corresponds to what is done experimentally and (2) it avoids problems related to air clusters on the electrodes. Indeed, injecting a fixed current would require a perfect knowledge of the wetting phase and solute concentration above the electrode, which is impossible with the present experimental setup.

\subsection{Petrophysical characterization}

Many petrophysical relationships have been proposed to relate electrical conductivity to porosity, water saturation, and electrolyte concentration (e.g., [6]). The experiments described in section 2.3 provided various data sets of measured electrical conductivities $\sigma^{\text {meas }}$ for different water saturations $S_{\mathrm{w}}$ at three different saline tracer conductivities $\sigma_{\mathrm{w}}$. Figures $4 \mathrm{a}$ and b show $\sigma^{\text {meas }}$ for different $\sigma_{\mathrm{w}}$ under saturated and partially saturated conditions, respectively.

Among the existing petrophysical relationships, let us consider the model that is the most used in the ERT literature: the classical model that is obtained by combining the so-called Archie's first and second law [50]. It is applicable when the mineral surface conductivity can be neglected (i.e., typically for materials with low specific surface area such as sands or sandstones):

where $\sigma$ and $\sigma_{\mathrm{w}}$ are the electrical conductivities $\left(\mathrm{S} \mathrm{m}^{-1}\right)$ of the porous medium and pore-water, respectively, $S_{\mathrm{w}}$ is the water saturation (-), $F$ is the electri- 


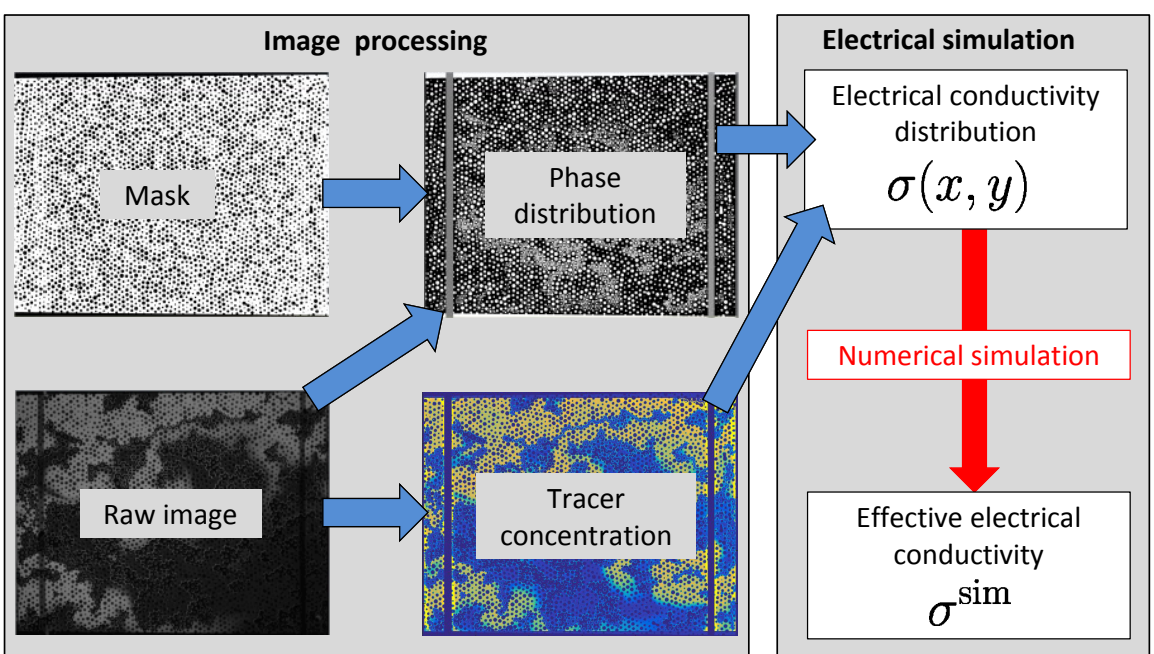

Figure 3: Schematic representation of the processing of the light-corrected images and the corresponding numerical simulation.

378 Equation (1) relating $\sigma$ and $\sigma_{\mathrm{w}}$ allows us to reproduce very well the experimental data in saturated conditions (Fig. 4a), but the data fit is not as good 380 for partially-saturated conditions (Fig. 4b). One likely reason for this is that 381 the sample size is too small to produce a representative elementary volume for 382

cal formation factor (-), and $n$ is the saturation exponent (-). The electrical formation factor is related to porosity by a power law: $F=\phi^{-m}$ where $m$ is the so-called cementation exponent. The parameters $m$ and $n$ depend on the pore-space and water phase geometry, respectively [e.g., Friedman 51]. The petrophysical parameters obtained by optimization, using the Simplex algorithm (Caceci and Cacheris [52]) and considering the entire dataset, are $F=$ 1.85 and $n=4$. the experiments with the lowest water saturations. 

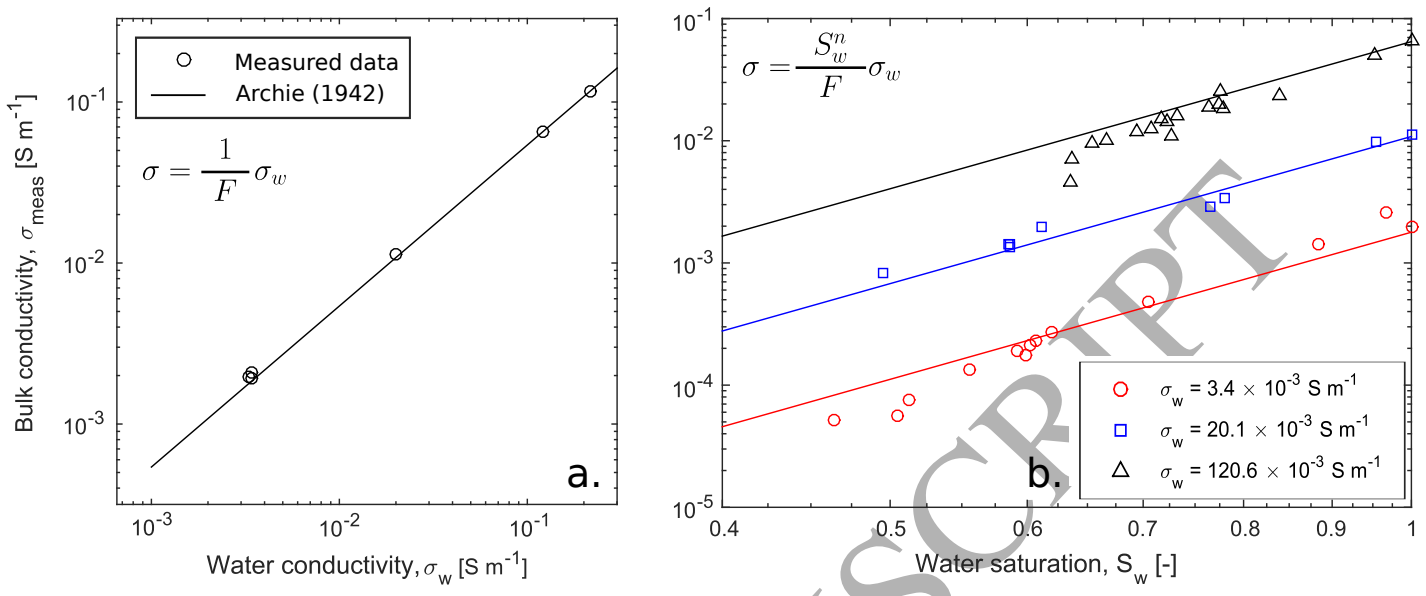

Figure 4: Bulk electrical conductivity as a function of (a) water conductivity in saturated conditions and (b) water saturation for different pore-water electrical conductivities. The dots (circles, squares, and triangles) correspond to the measurements (for different solutions) and the plain lines corresponds to the model of Archie [50]. The best fit to the entire data set was achieved with $F=1.85$ and $n=4$ in Eq. 1 . called Voigt and Reuss bounds.

The Wiener bounds are the arithmetic, $\bar{\sigma}_{\mathrm{w}}^{\mathrm{a}}$, and harmonic, $\bar{\sigma}_{\mathrm{w}}^{\mathrm{h}}$, means of the electrical conductivities $\sigma_{i}$ constituting the wetting phase:

$$
\bar{\sigma}_{\mathrm{w}}^{\mathrm{a}}=\frac{1}{N} \sum_{i=1}^{N} \sigma_{\mathrm{w}}^{i}
$$




$$
\bar{\sigma}_{\mathrm{w}}^{\mathrm{h}}=\frac{N}{\sum_{i=1}^{N} \frac{1}{\sigma_{\mathrm{w}}^{i}}},
$$

where $i$ denotes a single pixel belonging to the wetting phase and $N$ is the total number of pixels identified as the wetting phase. The arithmetic and harmonic means are equivalent to the global conductivity of an electrical circuit where conductances would be placed either in parallel or in series, respectively. The Wiener bounds theory predicts that:

$$
\bar{\sigma}_{\mathrm{w}}^{\mathrm{a}} \geq \sigma_{\mathrm{w}}^{\mathrm{app}} \geq \bar{\sigma}_{\mathrm{w}}^{\mathrm{h}}
$$

\section{Results} invades the medium. This increase is relatively smooth as a consequence of

${ }_{05}$ the tracer being transported according to an advection-diffusion process in a medium that is homogeneous at the Darcy scale. The tracer front is not perfectly straight nor transverse to the mean flow direction, due to some transverse 408 heterogeneity in the conditions of tracer injection at the inlet of the medium, 409 and possibly to a slight transverse permeability gradient in the medium. But no 410 solute fingers are visible at scales smaller than a third of the flow cell width, and 
homogeheously by the tracer $\left(\bar{\sigma}_{\mathrm{w}}^{\mathrm{a}} \neq \bar{\sigma}_{\mathrm{w}}^{\mathrm{h}}\right)$. Even with this homogeneous grain 436 distribution, some parts of the medium are left with comparatively low tracer concentrations (Fig. 5e).

\subsection{Tracer test under partially-saturated conditions}

The same procedure was applied to the data obtained from the two-phase 440

when the most advanced solute finger reaches the medium outlet, the most retarded region of the front has already reached half of the mediums length. Note that the rate of increase in the bulk electrical conductivity accelerates between $t=4000$ and $8000 \mathrm{~s}$ (Fig. 5a), which corresponds to the time interval over which increasing tracer concentration is in contact with both potential electrodes (P1 and P2). The rate of increase decreases after $t=8000 \mathrm{~s}$ when a continuous strip of highly concentrated tracer joins the two electrodes. This enables electrical current to flow through the cell with a lesser resistance.

After post-processing the images and solving the electrical problem at each time step (see section 3.1), it is possible to compare the measured bulk conductivity (i.e., flow cell scale, or macroscopic scale) to the simulated ones. Figure 6 a shows that the simulated conductivity $\left(\sigma^{\mathrm{sim}}\right)$ is in relatively good agreement with the data $\left(\sigma^{\text {meas }}\right)$ measured at the cell scale (root mean square error: $\left.R M S E=0.0021 \mathrm{~S} \mathrm{~m}^{-1}\right)$.

Figure $6 \mathrm{~b}$ shows the evolution of the apparent electrical conductivity of the wetting phase $\sigma_{\mathrm{w}}^{\text {app }}$ at the macroscopic scale (i.e., the scale of the flow cell) using Eq. 2. This value is compared to the arithmetic mean ("conductance in parallel" model) $\bar{\sigma}_{\mathrm{w}}^{\mathrm{a}}$ and to the harmonic mean ("conductance in series" model) $\bar{\sigma}_{\mathrm{w}}^{\mathrm{h}}$, showing that Eq. 5 is respected. Initially, $\sigma_{\mathrm{w}}^{\mathrm{app}}$ tends to follow the conductance in series model $\left(\bar{\sigma}_{\mathrm{w}}^{\mathrm{h}}\right)$ until fluid carrying a significant concentration of the tracer reaches the second potential electrode P2 (between $t=3000 \mathrm{~s}$ and $t=9000 \mathrm{~s})$. Then, $\sigma_{\mathrm{w}}^{\mathrm{app}}$ starts to follow the conductance in parallel model $\left(\bar{\sigma}_{\mathrm{w}}^{\mathrm{a}}\right)$ as the tracer tends to be distributed more homogeneously in the pore space. Note that at the end of the tracer test, the pore space is not filled ow tracer test. Figure 7a shows the evolution of the measured electrical con- 
$$
65
$$

$466 \quad 1$

that can be seen in Fig. $7 \mathrm{~d}$ (from $10^{-2}$ to $2 \times 10^{-2} \mathrm{~S} \mathrm{~m}^{-1}$ ). Fingering thus leads to strong increases in the electrical conductivity.

Figure $8 \mathrm{a}$ shows the comparison between the measured and the simulated electrical conductivities at the scale of the flow cell. The match between the simulation and the measurements, obtained with the same calibration curve 

have very slow kinetics (of order $D=10^{-10} \mathrm{~m}^{2} \mathrm{~s}^{-1}$ ) compared to the advec$l_{\text {air }} \simeq L / 4=40 \mathrm{~mm}$ as a typical length scale, the time necessary for the concentration to fully homogenize by ionic diffusion would be $l_{\text {air }}^{2} / D=1.6 \times 10^{7} \mathrm{~s}$ $500=185$ days. 

524 with a homogeneous solution concentration (Fig. 4a), we conducted numerical tests by calculating the bulk conductivity $\sigma^{\text {calc }}$ of only parts of the cell (not 526 shown here). These tests showed that the difference between the bulk electrical

\section{Discussion}

\subsection{Petrophysical characterization of the $2 D$ medium}

The petrophysical characterization of the analogous $2 \mathrm{D}$ porous medium is an important step for the interpretation of the present experiments. $F=1.85$ and $n=4$ can seem surprising for petrophysicists used to natural rocks. The low value of the formation factor is a consequence of the large porosity, and the high value of the exponent $n$ is the consequence of the $2 \mathrm{D}$ nature of the media under investigation (see the 2D pore network study of Maineult et al. [54]). Based on Archie [50], the electrical tortuosity of the wetting phase, $\alpha_{\mathrm{w}}$, can be defined as (Revil and Jougnot [55]):

$$
\alpha_{\mathrm{w}}=\phi F S_{\mathrm{w}}^{(1-n)}
$$

which yields $\alpha_{\mathrm{w}}=1.35$ and $\alpha_{\mathrm{w}}=4.04$ at the beginning of the saturated and partially saturated tracer tests, respectively. These small tortuosities can be visualized by considering the phase distributions (e.g., only 2D obstacles, long continuous path of wetting phase, see Figs. 5 and 7 ). Note that the electrical tortuosity of the water phase determined by electrical conductivity measurements can be used to predict different transport properties of interest in hydrology, such as the ionic and gas diffusion coefficients (e.g., [55, 56, 57]) or thermal conductivity (e.g., [58, 59]).

Figure 4 shows that the fit of Archie's model for data obtained at full saturation is better than the fit obtained under partially saturated conditions. This can be explained by considering the concept of Representative Elementary Volume (REV): the smallest volume over which a measurement can be made to obtain a value representative of the whole $([60])$. For saturated conditions conductivity calculated over a quarter of the cell and over the entire domain was smaller than $1.26 \%$. Thus demonstrating that the REV of the saturated 

ifest itself in simulated electrical current densities in saturated (Fig. 9c) and partially-saturated (Fig. 9d) conditions. In the fully saturated media, we find a 554 current density that is homogeneously-distributed in the pore space, while in the

medium $R E V^{\text {sat }}$ is smaller than the flow cell. However, substantial differences between sub-domains occurred when we conducted a similar numerical study on the partially saturated medium. Therefore, the size of REV for the partially saturated medium, $R E V^{\text {unsat }}$ is likely larger than the size of the cell itself. This is expected as the linear size of the largest air clusters in the medium is of about half of the medium size. Consequently, we illustrate here the following inequality:

$$
R E V^{\mathrm{sat}} \ll R E V^{\mathrm{unsat}} .
$$

This is well-known in the literature (e.g., [61]), but it is not always accounted for in hydrogeophysical studies. The limitations of Archie's law in the presence of percolation phenomena are discussed, for instance, by Kozlov et al. [43].

\subsection{Relationship between the concentration field and the measured conductivity}

As shown in section 4, the heterogeneity of the tracer concentration field strongly depends on the saturation of the medium. Indeed, the concentration field is much more heterogeneous under partially saturated conditions than in the saturated case. As seen in Fig. 7d, at the end of the experiment ( $t=14000 \mathrm{~s})$, large parts of the cell are still at the background solution concentration and the tracer is far from being homogeneously distributed. Note that molecular/ionic diffusion effects will eventually homogenize the tracer concentration but with very slow kinetics (see discussion in [35]).

Figure 9 compares the normalized tracer concentration distribution at a late stage of saturated and partially-saturated tracer tests and the corresponding simulated electrical current densities. The impact of the saturation distribution has a strong effect on the tracer distribution (Figs. 9a and b) that clearly manpartially-saturated case it is highly channelized. We find that the current paths are straighter and less tortuous than the saline tracer distribution (Figs. 9c and 
$$
\text { 尘 }
$$

582

2 con

The dynamics and evolution of the effective tracer percolation through the medium are also well captured by the bulk electrical conductivity. In saturated

d). As expected, while the saturated porous media shows a homogeneous distribution of current densities, the unsaturated porous media is characterized by a highly channelized current density field.

In our experimental results, we show that the effective bulk electrical conductivity at the scale of the flow cell carries information about transport processes occurring at smaller scales. Electrical conductivity is sensitive to the saline tracer distribution in the medium. Indeed, for both saturated and partiallysaturated conditions, a very strong increase of the effective bulk electrical conductivity can be seen when the saline tracer connects electrodes $\mathrm{P} 1$ and $\mathrm{P} 2$ (Figs. 5 and 7). The connectivity of the tracer in the medium is illustrated by the Wiener bounds; the apparent water conductivity is initially analogous to a model of conductance in series before it starts to follow a model of conductances in parallel. This analysis is even more instructive in partially saturated conditions as the breakthrough of only one solute finger through the medium acts as a preferential path for the electrical current, yielding a very strong change in bulk electrical conductivity.

Tracer fingers in the unsaturated case act as preferential paths for the electrical current (Fig. 9d) in comparison to the more homogeneous case in saturated conditions (Fig. 9b). One can identify "bottle-necks" as the places where the flow lines are focused between air clusters or grains (e.g., [35]). This effect on the tracer transport affect the distribution of the electrical current flow (Fig. 9d). This channeling of the electrical current density is not only controlled by the tracer concentration alone, but also by how a region containing high tracer concentrations connects the different electrodes of the measurement setup. For example, the large tracer concentration area in the middle of Fig. 9d does not ntribute significantly to the electrical flow. conditions, the smooth increase of $\sigma$ during the entire tracer test corresponds to the progressive invasion of the flow cell by the saline tracer (Fig. 5). On the contrary, the more abrupt increase of $\sigma$ around $t=4000 \mathrm{~s}$ and the smaller 


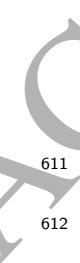

613

614

one around $t=9000 \mathrm{~s}$ (Fig. 7) denote the percolation of the first and second fingers of tracer through the porous medium. This clear link between tracer percolation and bulk electrical conductivity should be studied in more details to couple transport models at the pore-scale and hydrogeophysical measurements, for example following the idea proposed by Kemna et al. [9] at the field-scale.

\subsection{Electrical conductivity anisotropy}

From the previous subsection, it appears that the bulk electrical conductivity is strongly related to the liquid phase connectivity and to the spatial distribution of the tracer concentration. It is therefore highly sensitive to the orientation of the electrical conductivity measurement setup with respect to the tracer transport. In order to study this effect, numerical tests have been performed: for each time step, the simulated bulk electrical conductivity presented in sections 4.1 and 4.2 (the longitudinal bulk electrical conductivity) has been compared to the value obtained from a numerical simulation where the current flows in the medium from top to bottom, so that we compute the transverse bulk electrical conductivity. That is, we extracted the section of the images between electrode P1 and P2 (i.e., the investigation zone) and solved the electrical problem after imposing a $90^{\circ}$ rotation to the porous medium around its center, without modifying the position of the electrodes. Figures 10a and b illustrate the simulation set-up of this anisotropy study, while Fig. 10c and d show the anisotropy factor $\lambda$ of the electrical conductivity during the saturated and the partially saturated tracer tests, respectively. The dimensionless anisotropy factor, $\lambda$, is calculated as follows [e.g. 62]:

$$
\lambda=\sqrt{\frac{\sigma^{\operatorname{sim}}}{\sigma_{90}^{\operatorname{sim}}}},
$$

where $\sigma^{\text {sim }}$ and $\sigma_{90}^{\text {sim }}$ are the longitudinal and the transverse electrical conductivities with respect to the fluid flow direction, respectively. Finally, Figures 10e and $\mathrm{f}$ are conceptual illustrations of ERT measurements for a lateral or vertical tracer test in the near surface, respectively. 


\section{0}

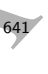

642

643 644

The behaviour of the electrical anisotropy factor is completely different under saturated and partially saturated conditions (Fig. 10). Under saturated conditions (Fig. 10b), the bulk electrical conductivity of the medium is isotropic (i.e., $\lambda \simeq 1$ ) at the beginning and at the end of the tracer test. During the tracer test, the transverse electrical conductivity is higher than the longitudinal one as the zone that is invaded by the tracer create a high conductivity path for the electrical current from top to bottom (Fig. 5). On the contrary, under partially saturated, the medium is already anisotropic due to the presence of air clusters, the largest of which have a linear size close to half the medium size. Given the flow direction in the cell, air clusters tend to be more elongated in the longitudinal direction (Fig. 7), which yield $\sigma^{\text {sim }}>\sigma_{90}^{\text {sim }}(\lambda=1.15$ for $t=0 \mathrm{~s})$. Then, as the tracer propagates in the pore space, the complex patterns of tracer fingering tend to increase the anisotropy factor (up to $\lambda=2.41$ ). Note that, at the end of the tracer test, the anisotropy factor diminishes but does not return to its initial value (e.g. $\lambda=1.59$ at $t=14000 \mathrm{~s}$ ). This is caused by the remaining strong heterogeneity in the tracer concentration distribution (Fig. 9).

\subsection{Impact on ERT interpretations}

This study presents a new geoelectrical milli-fluidic setup that helps understanding better the link between state variables of hydrogeological interest (water saturation, flow velocity field, ionic concentration field, solute concentration field) and measurable upscaled geophysical properties under dynamic conditions. Considering that the scale of the flow cell in our experiment could be seen as an analogue to the resolution scale in a field ERT experiment, the present work has clearly shown that the apparent water electrical conductivity at the resolution scale is largely determined by the heterogeneity of ionic concentrations below this resolution scale, especially under partially saturated conditions (see Figs. 5 to 9).

Electrical conductivities resulting from ERT inversion are subjected to two kinds of processes masking the medium heterogeneity: (1) a smoothing above the cell size used to discretize the subsurface that is due to the inversion reg- 

the two effects described above (regularization and homogenization). However, in section 4 , we have shown that the bulk electrical conductivity measured at 673 the scale of our porous medium depends strongly on the connectivity of the

ularization imposed to make the inverse problem unique and (2) the conductivity homogenization at the REV (or discretized cell size) scale that we study herein. The inversion smoothing is the most scrutinized one as it is due to a regularization process needed to perform the inversion [e.g. Constable et al. 22]. Various works have described and studied the impact of model regularization, showing that information about sharp contrasts between model cells is lost (see, among other works: Day-Lewis et al. [18], Singha and Gorelick [19]). This phenomenon induces an apparent tracer loss when conducting in situ ERT monitoring of tracer tests. The second process masking the heterogeneity of the processes at play is a consequence of unaccounted saline tracer heterogeneity below the cell size used in the inversion. This cell size is often implicitly assumed to correspond to the REV scale and petrophysical models are used (see Eq. 1) that assumes that saline tracer heterogeneity is constant. Only a few works have considered tracer heterogeneity at the REV scale (e.g., [23], [28]), but only for two classes of porosities. Future work is needed to ensure proper upscaling to the cell size used in geophysical inversion and forward modeling. This work has clearly demonstrated that such effects can be very strong. In practice, we suggest that both of these effects are interconnected and we refer to them collectively as sub-resolution effects.

In our experiments we find that the heterogeneous fluid phase distributions and ionic concentration field in the aqueous phase at the sub-resolution scale have shown a strong impact on the effective bulk electrical conductivity. When conducting ERT monitoring, researchers often try to retrieve the ionic concentration at the resolution scale from the bulk electrical conductivity using a petrophysical relationship (often Archie [50], Eqs. 1 and 2) which assume a homogeneous distribution of $\sigma_{\mathrm{w}}$ below the resolution scale. Thus, they ignore tracer between the two potential electrodes at the pore-scale. Figures $6 \mathrm{~b}$ and $8 \mathrm{~b}$ show that as long as the medium is not homogeneous, the apparent water 

2 taking advantage of this anisotropy.

Finally, to the best of our knowledge, the electrical conductivity has never 704 been considered a tensor in time-lapse ERT inversions [see 63, 64, for static in-

conductivity can be much smaller than the arithmetic mean of its constituents: $\sigma_{\mathrm{w}}^{\mathrm{app}} \ll \bar{\sigma}_{\mathrm{w}}^{\mathrm{a}}$. Indeed, the electrical current is only sensitive to a fraction of the tracer in the medium, the part connecting both sides of the voxel in the direction of the electrical current, as shown by Fig. 9. And, as discussed previously, the time of homogeneization can be much longer than the duration of an experiment (be it a lab or a field experiment). This inequality between effective bulk conductivity and arithmetic average conductivity results in an apparent mass loss when inferring solute concentrations from ERT inversion results using Eq. (2). Indeed, in our medium, the arithmetic mean of the local conductivities (the upper Wiener bound), $\bar{\sigma}_{\mathrm{w}}^{\mathrm{a}}$, is proportional to the arithmetic mean of local concentrations, which is the solute concentration defined at the medium scale. If one considers that our medium scale represents the resolution scale of an ERT field experiment (Figs. 10e and f), the solute concentration measured by ERT would be inferred from an apparent water conductivity measured at that scale, and hence its ratio to the true solute concentration would equal the ratio $\sigma_{\mathrm{w}}^{\mathrm{app}} / \bar{\sigma}_{\mathrm{w}}^{\mathrm{a}}$. In other words the apparent mass loss is a necessary consequence of the upper Wiener bound at sub-resolution scale and is to be expected as soon as the solute is not well-mixed in the pore space and at the ERT resolution scale (i.e., always). This mechanism could account for an important proportion of the very important mass loss in ERT studies (e.g., $75 \%$ of apparent mass loss in Singha and Gorelick [10]). Furthermore, these results indicate that monitoring an horizontal tracer flow (Fig. 10e) or a vertical one (Fig. 10f) from the surface could result in very different apparent mass losses, depending also on the type of ERT measurements performed (surface measurements or borehole measurements). The use of electrode arrays at both the surface and in boreholes [e.g. 14], when available, could help better constraining the tracer flow direction by

version]. Therefore, the effect of tracer percolation on the electrical conductivity anisotropy factor is largely ignored and will inevitably produce artifacts. Given 
the large value obtained in the anisotropy test (up to $\lambda=2.76$ in Fig. 10d), it seems that describing electrical conductivity as a tensorial property during tracer tests should be investigated in more details.

\subsection{Technical improvements for further experiments}

We have developed a geoelectrical milli-fluidic setup to study sub-resølution effects associated with electrical conductivity monitoring of tracer tests. Even if this initial study is already rich in results, significant improvements could be made to the experimental setup and measurement protocol.

The opacity of the currently used electrodes has the disadvantage of degrading the information available about what is happening above them (Figs. 5,7 , and 9), making for example the determination of air cluster boundaries above an electrode difficult. Different possibilities can be explored to solve this issue, among which the use of transparent electrodes, or locating the electrodes outside of the porous medium.

An appropriate choice of fluorescein concentration in the injected solutions is crucial to obtain high-quality images with a suitable optical contrast. For future works, we recommend that the background solution should be more concentrated in fluorescein, in order to improve the contrast between the liquid phase at low fluorescein concentration and the air phase. Furthermore, the mass concentration ratio between fluorescein and $\mathrm{NaCl}$ salt should be revisited to take adyantage of the largest range of light intensity possible. We anticipate that performing the calibration of the transfer function between light intensity and local conductivity in the same flow cell as that used for the tracer experiments will remove the need for an a posteriori correction of the calibration curve. Besides, the use of many more calibration points will help improving the reliability of that curve.

\section{Conclusion}

We propose a new geoelectrical milli-fluidic setup to study the impact of the pore-scale distribution of fluid phases and tracer concentrations on the effective 


\section{Acknowledgment}

The authors gratefully acknowledge support from the EC2CO program of INSU/CNRS (project AO2014-906387). The experimental work was also supported by the Interreg project CLIMAWAT, EU-RDF INTERREG IVA France 762 (Channel)-England program. The data used are available by contacting the 763 764

bulk electrical conductivity. The setup is based on a two-dimensional porous medium consisting of a single layer of cylindrical grains positioned randomly between two parallel glass plates. The macroscopic scale in our laboratory study can be seen as an analogue to the resolution scale of ERT at the field-scale. After performing a petrophysical characterization of the porous medium, we monitored variations in the bulk electrical conductivity of the porous medium during saline tracer tests under full and partial saturation. We find that the air distribution and the resulting heterogeneity in solute concentrations lead to electrical current channeling with strong effects on effective bulk conductivities. This suggests a strong impact of pore-scale and sub-resolution effects on upscaled bulk electrical conductivity in terms of magnitude and anisotropy. Such effects are expected to systematically occur at the field-scale due to incomplete solute mixing below the resolution scale. We suggest that they could contribute significantly to an important inconsistency in conventional time-lapse ERT image processing, namely the apparent loss of tracer mass. In fact, the use of the upper Wiener bound of the effective bulk electrical conductivity implies that the inferred solute concentration at the ERT resolution scale will always be smaller than the true average solute concentration at that scale. The presented geoelectrical milli-fluidic setup (and variations thereof) opens up a range of opportunities to investigate the link between electrical signals and a variety of pore-scale processes, such as mixing, reactive transport, and biogeochemical reactions

corresponding author. The authors gratefully thank the editor, Kamini Singha, and an anonymous reviewer for their very constructive comments. 
[9] A. Kemna, B. Kulessa, H. Vereecken, Imaging and characterisation of subsurface solute transport using electrical resistivity tomography (ERT) and equivalent transport models, Journal of Hydrology 267 (3) (2002) 125-146.

[10] K. Singha, S. M. Gorelick, Saline tracer visualized with three-dimensional

\section{References}

\section{References}

[1] S. S. Hubbard, Y. Rubin, Introduction to hydrogeophysics, in: Hydrogeophysics, Springer, 2005, pp. 3-21.

[2] R. Guérin, Borehole and surface-based hydrogeophysics, Hydrogeology Journal 13 (1) (2005) 251-254.

[3] A. Binley, G. Cassiani, A. Revil, K. Titov, H. Vereecken, Applied Hydrogeophysics, Springer, 2006.

[4] S. Hubbard, N. Linde, Hydrogeophysics, in: P. Wilderer (Ed.), Treatise on Water Science, Vol. 1, Oxford: Academic Press, 2011,pp. 401-434.

[5] A. Binley, S. S. Hubbard, J. A. Huisman, A. Revil, D. A. Robinson, K. Singha, L. D. Slater, The emergence of hydrogeophysics for improved understanding of subsurface processes over multiple scales, Water Resources Research 51 (6) (2015) 3837-3866.

[6] P. Glover, Geophysical properties of the near surface earth: electrical properties, Treatise on Geophysics 11 (2015) 89-137.

[7] A. Binley, A. Kemna, Dc resistivity and induced polarization methods, in: Hydrogeophysics, Springer, 2005, pp. 129-156.

[8] A. Revil, M. Karaoulis, T. Johnson, A. Kemna, Review: Some lowfrequency electrical methods for subsurface characterization and monitoring in hydrogeology, Hydrogeology Journal (2012) 1-42. electrical resistivity tomography: Field-scale spatial moment analysis, Water Resources Research 41 (5) (2005) W05023. 
[11] D. Pollock, O. A. Cirpka, Fully coupled hydrogeophysical inversion of a laboratory salt tracer experiment monitored by electrical resistivity tomography, Water Resources Research 48 (1) (2012) W01505.

[12] W. Daily, A. Ramirez, D. LaBrecque, J. Nitao, Electrical resistivity tomography of vadose water movement, Water Resources Research 28 (5) (1992) 1429-1442.

[13] A. Binley, G. Cassiani, R. Middleton, P. Winship, Vadose zone flow model parameterisation using cross-borehole radar and resistivity imaging, Journal of Hydrology 267 (3) (2002) 147-159.

[14] M. C. Looms, K. H. Jensen, A. Binley, L. Nielsen, Monitoring unsaturated flow and transport using cross-borehole geophysical methods, Vadose Zone Journal 7 (1) (2008) 227-237.

[15] E. B. Haarder, K. H. Jensen, A. Binley, L. Nielsen, T. Uglebjerg, M. Looms, Estimation of recharge from long-term monitoring of saline tracer transport using electrical resistivity tomography, Vadose Zone Journal 14 (7) (2015) $1-13$.

[16] W. Menke, Geophysical Data Analysis: Discrete Inverse Theory, International Geophysics Series, New York: Academic Press, 1989, Rev. ed. 1.

[17] S. Friedel, Resolution, stability and efficiency of resistivity tomography estimated from a generalized inverse approach, Geophysical Journal International 153 (2) (2003) 305-316.

[18] F. D. Day-Lewis, K. Singha, A. M. Binley, Applying petrophysical models to radar travel time and electrical resistivity tomograms: Resolutiondependent limitations, Journal of Geophysical Research: Solid Earth 110 (B8) (2005) B08206.

[19] K. Singha, S. M. Gorelick, Effects of spatially variable resolution on fieldscale estimates of tracer concentration from electrical inversions using archies law, Geophysics 71 (3) (2006) G83-G91. 


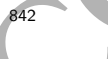

$843 \quad[27]$

[20] M. Rosas-Carbajal, N. Linde, J. Peacock, F. Zyserman, T. Kalscheuer, S. Thiel, Probabilistic 3-d time-lapse inversion of magnetotelluric data: application to an enhanced geothermal system, Geophysical Journal International 203 (3) (2015) 1946-1960.

[21] J. Doetsch, N. Linde, T. Vogt, A. Binley, A. G. Green, Imaging and quantifying salt-tracer transport in a riparian groundwater system by means of 3D ERT monitoring, Geophysics 77 (5) (2012) B207-B218.

[22] S. C. Constable, R. L. Parker, C. G. Constable, Occam's inversion: A practical algorithm for generating smooth models from electromagnetic sounding data, Geophysics 52 (3) (1987) 289-300.

[23] K. Singha, F. D. Day-Lewis, J. Lane, Geoelectrical evidence of bicontinuum transport in groundwater, Geophysical Research Letters 34 (12).

[24] K. Singha, A. Pidlisecky, F. D. Day-Lewis, M. N. Gooseff, Electrical characterization of non-fickian transport in groundwater and hyporheic systems, Water Resources Research 44 (4) (2008) W00D07.

[25] R. D. Swanson, A. Binley, K. Keating, S. France, G. Osterman, F. D. DayLewis, K. Singha, Anomalous solute transport in saturated porous media: Relating transport model parameters to electrical and nuclear magnetic resonance properties, Water Resources Research 51 (2) (2015) 1264-1283.

[26] M. A. Briggs, F. D. Day-Lewis, J. B. Ong, G. P. Curtis, J. W. Lane, Simultaneous estimation of local-scale and flow path-scale dual-domain mass transfer parameters using geoelectrical monitoring, Water Resources Research 49 (9) (2013) 5615-5630.

M. A. Briggs, F. D. Day-Lewis, J. B. Ong, J. W. Harvey, J. W. Lane, Dual-domain mass-transfer parameters from electrical hysteresis: Theory and analytical approach applied to laboratory, synthetic streambed, and groundwater experiments, Water Resources Research 50 (10) (2014) 82818299. 
[34] S. M, Hassanizadeh, M. A. Celia, H. K. Dahle, Dynamic effect in the capillary pressure-saturation relationship and its impacts on unsaturated flow, Vadose Zone Jounal 1 (1) (2002) 38-57.

[35] J. Jiménez-Martínez, P. de Anna, H. Tabuteau, R. Turuban, T. L. Borgne, Y. Méheust, Pore-scale mechanisms for the enhancement of mixing in un874 saturated porous media and implications for chemical reactions, Geophys875

[28] F. D. Day-Lewis, N. Linde, R. Haggerty, K. Singha, M. A. Briggs, Pore network modeling of the electrical signature of solute transport in dualdomain media, Geophysical Research Letters 44 (10) (2017) 4908-4916, 2017 GL073326.

[29] R. Glass, M. Nicholl, Physics of gravity fingering of immiscible fluids within porous media: An overview of current understanding and selected complicating factors, Geoderma 70 (2-4) (1996) 133-163.

[30] Y. Méheust, G. Løvoll, K. J. Måløy, J. Schmittbuhl, Interface scaling in a two-dimensional porous medium under combined viscous, gravity, and capillary effects, Physical Review E 66 (5) (2002) 051603.

[31] R. Toussaint, K. J. Må løy, K., Y. Méheust, G. Løvoll, M. Jankov, G. Schäfer, J. Schmittbuhl, Two-phase flow: structure, upscaling, and consequences for macroscopic transport properties, Vadose Zone Journal $11(3)$.

[32] A. Ferrari, I. Lunati, Direct numerical simulations of interface dynamics to link capillary pressure and total surface energy, Advances in Water Resources 57 (2013) 19-31.

[33] G. Løvoll, M. Jankov, K. J. Måløy, R. Toussaint, J. Schmittbuhl, G. Schäfer, Y. Méheust, Influence of viscous fingering on dynamic saturation-pressure curves in porous media, Transport in Porous Media 86 (2011) 305-324. ical Research Letters 42 (13) (2015) 5316-5324. 


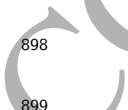

\section{[43]}

[36] J. Jiménez-Martínez, T. Le Borgne, H. Tabuteau, Y. Méheust, Impact of saturation on dispersion and mixing in porous media: Photobleaching pulse injection experiments and shear-enhanced mixing model, Water Resources Research 53 (2017) 1457-1472.

[37] M. Dentz, T. Le Borgne, A. Englert, B. Bijeljic, Mixing, spreading and reaction in heterogeneous media: A brief review, Journal of Contaminant Hydrology 120 (2011) 1-17.

[38] T. Le Borgne, M. Dentz, P. Davy, D. Bolster, J. Carrera, J.-R. De Dreuzy, O. Bour, Persistence of incomplete mixing: A key to anomalous transport, Physical Review E 84 (1) (2011) 015301.

[39] M. Seyfried, P. Rao, Solute transport in undisturbed columns of an aggregated tropical soil: Preferential flow effects, Soil Science Society of America Journal 51 (6) (1987) 1434-1444.

[40] J. Koestel, M. Larsbo, Imaging and quantification of preferential solute transport in soil macropores, Water Resources Research 50 (5) (2014) 43574378.

[41] T. W. Willingham, C. J. Werth, A. J. Valocchi, Evaluation of the effects of porous media structure on mixing-controlled reactions using pore-scale modeling and micromodel experiments, Environmental science \& technology 42 (9) (2008) 3185-3193.

[42] P. de Anna, J. Jimenez-Martinez, H. Tabuteau, R. Turuban, T. Le Borgne, M. Derrien, Y. Méheust, Mixing and reaction kinetics in porous media: An experimental pore scale quantification, Environmental Science \& Technology 48 (1) (2013) 508-516.

3] B. Kozlov, M. Schneider, B. Montaron, M. Lagues, P. Tabeling, Archie's law in microsystems, Transport in Porous Media (2012) 1-20. 
[44] A. Ferrari, J. Jimenez-Martinez, T. L. Borgne, Y. Méheust, I. Lunati, Challenges in modeling unstable two-phase flow experiments in porous micromodels, Water Resources Research 51 (3) (2015) 1381-1400.

[45] R. Sjöback, J. Nygren, M. Kubista, Absorption and fluorescence properties of fluorescein, Spectrochimica Acta Part A: Molecular and Biomolecular Spectroscopy 51 (6) (1995) L7-L21.

[46] C. Schlumberger, Etude sur la prospection electrique du sous-sol [Study on underground electrical prospecting], Gauthier-Villars, 1920.

[47] D. Jougnot, A. Ghorbani, A. Revil, P. Leroy, P. Cosenza, Spectral induced polarization of partially saturated clay-rocks: A mechanistic approach, Geophysical Journal International 180 (1) (2010) 210-224.

[48] K. T. Tallakstad, H. A. Knudsen, T. Ramstad, G. Løvoll, K. J. Måløy, R. Toussaint, E. G. Flekkøy, Steady-State Two-Phase Flow in Porous Media: Statistics and Transport Properties, Physical Review Letters 102 (2009) 074502.

[49] R. Künze, I. Lunati, An adaptive multiscale method for density-driven instabilities, Journal of Computational Physics 231 (17) (2012) 5557-5570.

[50] G. Archie, The electrical resistivity log as an aid in determining some reservoir characteristics, Transaction of the American Institute of Mining and Metallurgical Engineers 146 (1942) 54-61.

[51] S. P. Friedman, Soil properties influencing apparent electrical conductivity: a review, Computers and electronics in agriculture 46 (1) (2005) 45-70.

[52] M. Caceci, W. Cacheris, Simplex optimization algorithm, Byte 5 (1984) $340-351$.

[53] O. Wiener, Theory of composite bodies, Abh. sächs. Ges. Wiss. 33 (1912) $507-525$. 
[54] A. Maineult, D. Jougnot, A. Revil, Variations of petrophysical properties and spectral induced polarization in response to drainage and imbibition: a study on a correlated random tube network, submitted to Geophysical Journal International.

[55] A. Revil, D. Jougnot, Diffusion of ions in unsaturated porous materials, Journal of Colloid and Interface Science 319 (1) (2008) 226-235.

[56] D. Jougnot, A. Revil, P. Leroy, Diffusion of ionic tracers in the calløvooxfordian clay-rock using the donnan equilibrium model and the formation factor, Geochimica et Cosmochimica Acta 73 (10) (2009) 2712-2726.

[57] S. Hamamoto, P. Moldrup, K. Kawamoto, T. Komatsu, Excluded-volume expansion of Archie's law for gas and solute diffusivities and electrical and thermal conductivities in variably saturated porous media, Water Resources Research 46 (6).

[58] A. Revil, Thermal conductivity of unconsolidated sediments with geophysical applications, Journal of Geophysical Research 105 (B7) (2000) 1674916 .

[59] D. Jougnot, A. Revil, et al., Thermal conductivity of unsaturated clayrocks, Hydrology and Earth System Sciences 14 (1) (2010) 91-98.

[60] R. Hill, Elastic properties of reinforced solids: Some theoretical principles, Journal of the Mechanics and Physics of Solids 11 (5) (1963) 357 - 372.

[61] J. Joekar-Niasar, S. M. Hassanizadeh, Specific interfacial area: The missing state yariable in two-phase flow equations?, Water Resources Research 47 (2011) W05513.

[62] N. Linde, L. B. Pedersen, Evidence of electrical anisotropy in limestone formations using the RMT technique, Geophysics 69 (4) (2004) 909-916.

[63] D. J. LaBrecque, D. Casale, Experience with anisotropic inversion for electrical resistivity tomography, in: 15th EEGS Symposium on the Application of Geophysics to Engineering and Environmental Problems, 2002. 
${ }_{956}[64]$ C. C. Pain, J. V. Herwanger, J. H. Saunders, M. H. Worthington, C. R.

957 de Oliveira, Anisotropic resistivity inversion, Inverse Problems 19 (5) (2003)

$958 \quad 1081$.

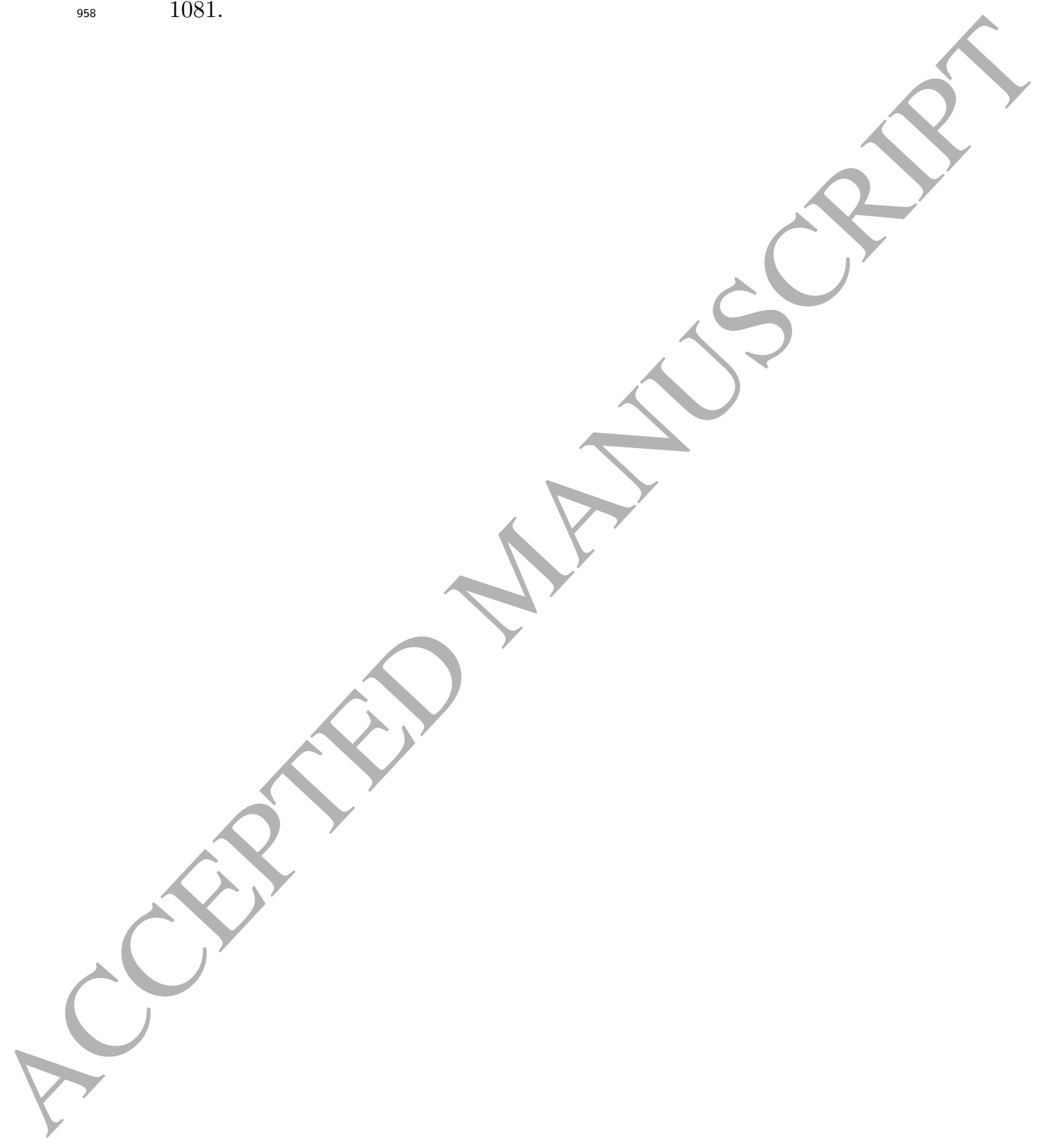



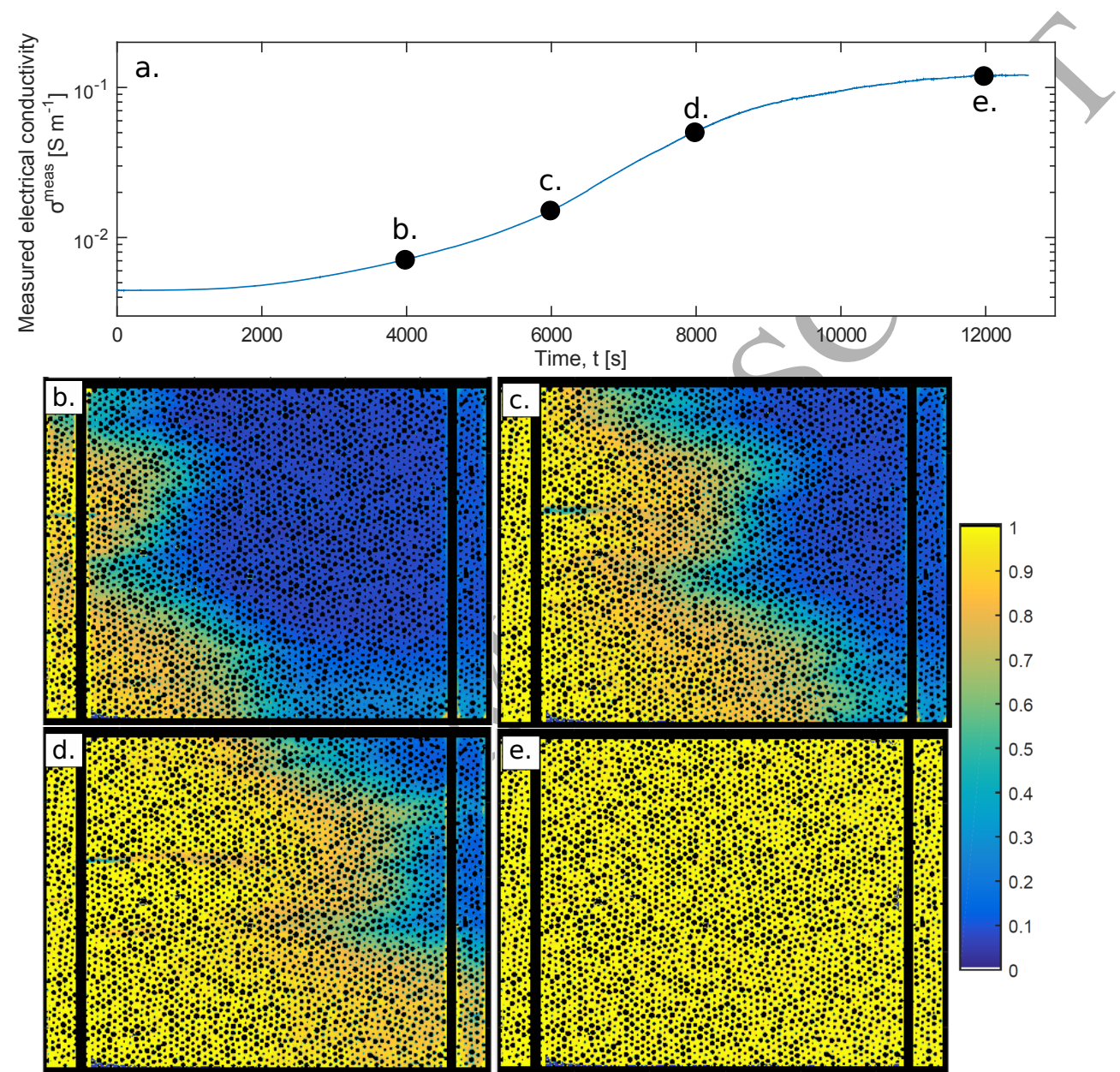

Figure 5: (a) Measured effective bulk electrical conductivity as a function of the time since the initiation of a tracer test in saturated conditions and corresponding images of the normalized tracer concentration in the flow cell at (b) $t=4000 \mathrm{~s}$, (c) $t=6000 \mathrm{~s}$, (d) $t=8000 \mathrm{~s}$, and (b) $t=12000 \mathrm{~s}$. The grains, the top and bottom boundaries, and the four electrodes appear as either black lines or circles on the image. 

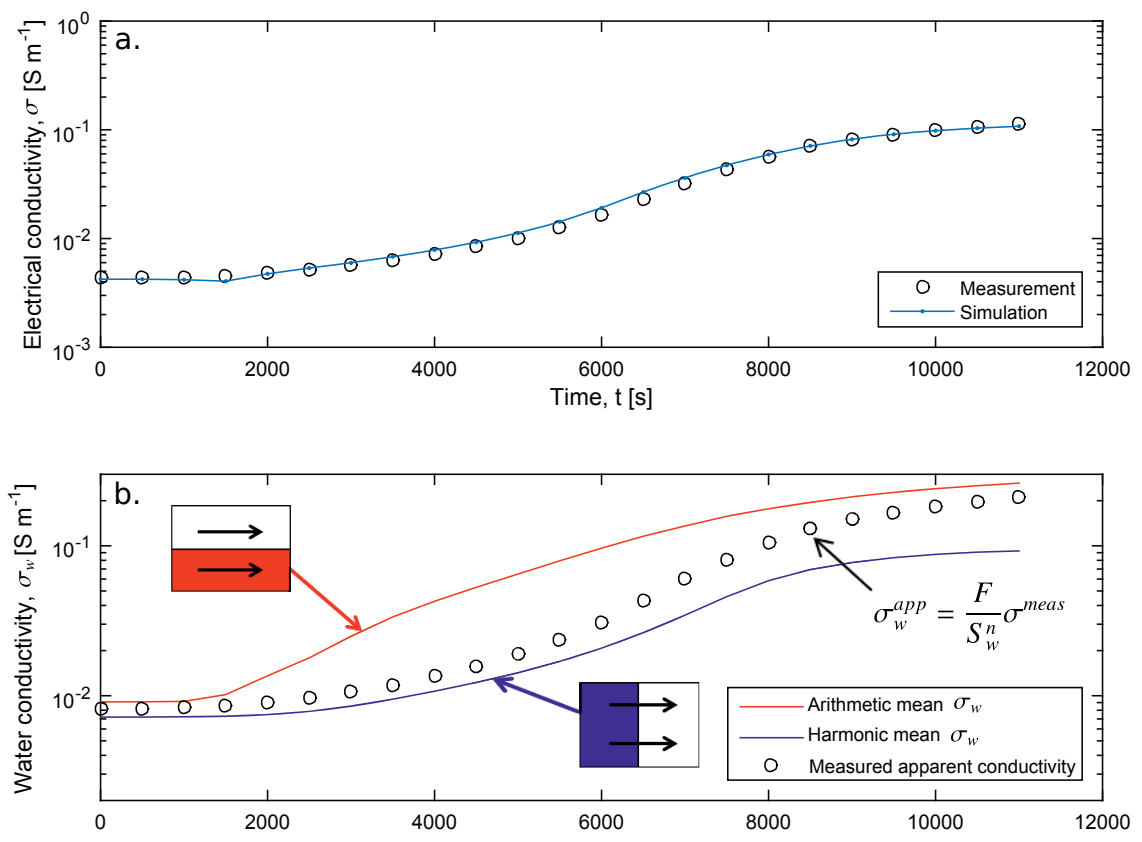

Figure 6: (a) Comparison between measured and simulated electrical conductivities at the scale of the flow cell under saturated conditions $\left(R M S E=0.0021 \mathrm{~S} \mathrm{~m}^{-1}\right)$. (b) Apparent water electrical conductivity from Eq. 2 (with $F=1.85$ ) and Wiener bound values of the wetting phase at the flow cell scale. 

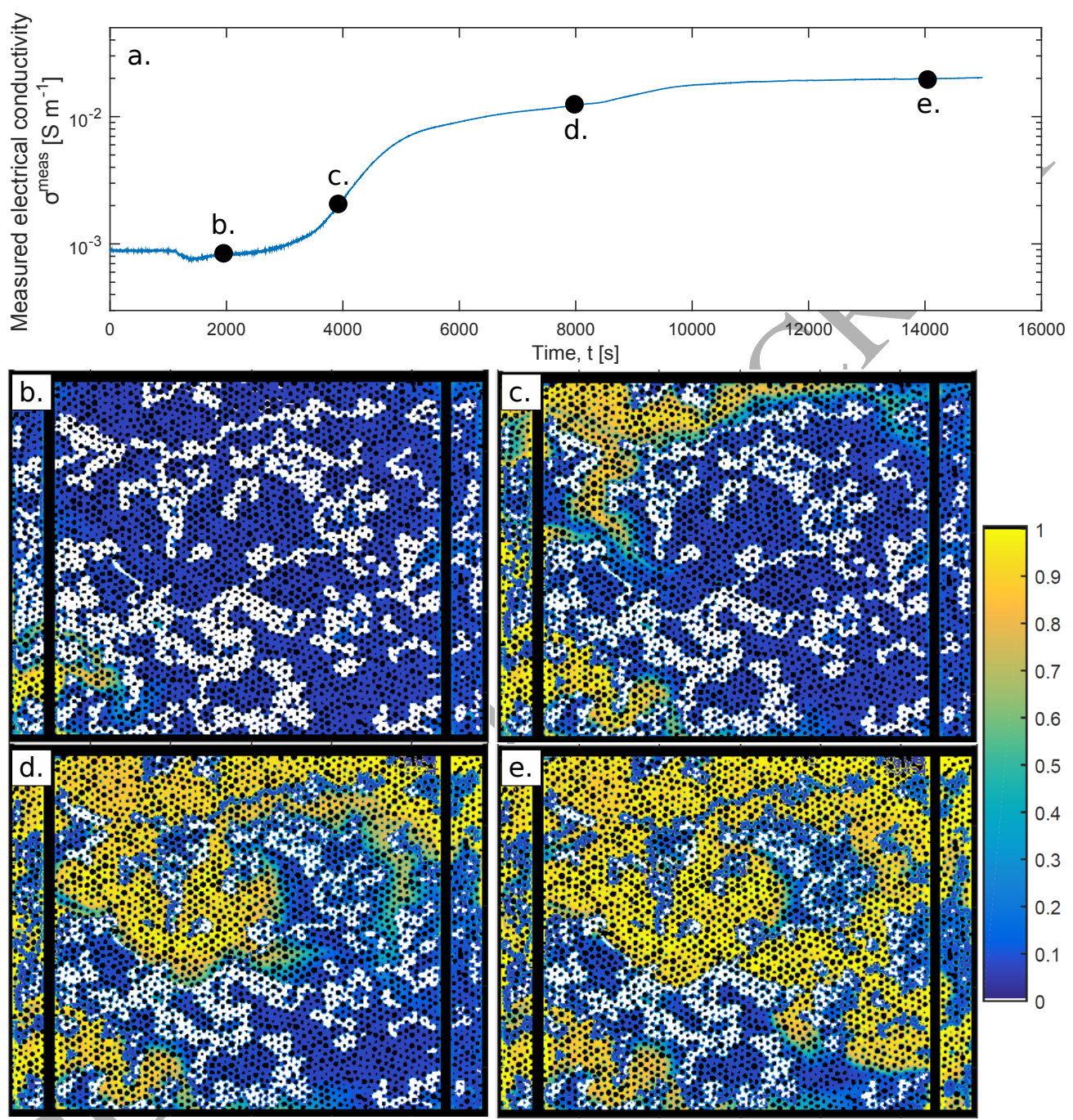

Figure 7: (a) Measured effective bulk electrical conductivity as a function of the time during a tracer test in partially saturated conditions and corresponding images of the normalized tracer concentration in the test cell at (b) $t=2000 \mathrm{~s}$, (c) $t=4000 \mathrm{~s}$, (d) $t=8000 \mathrm{~s}$, and (e) $t=14000 \mathrm{~s}$. The grains, the top and bottom boundaries, and the four electrodes appear as either black lines or circles on the image. The air appears in white clusters since there is no fluorescein in this phase. During the course of the experiment, the water saturation increased from $S_{\mathrm{w}}=0.69$ to 0.87 . 

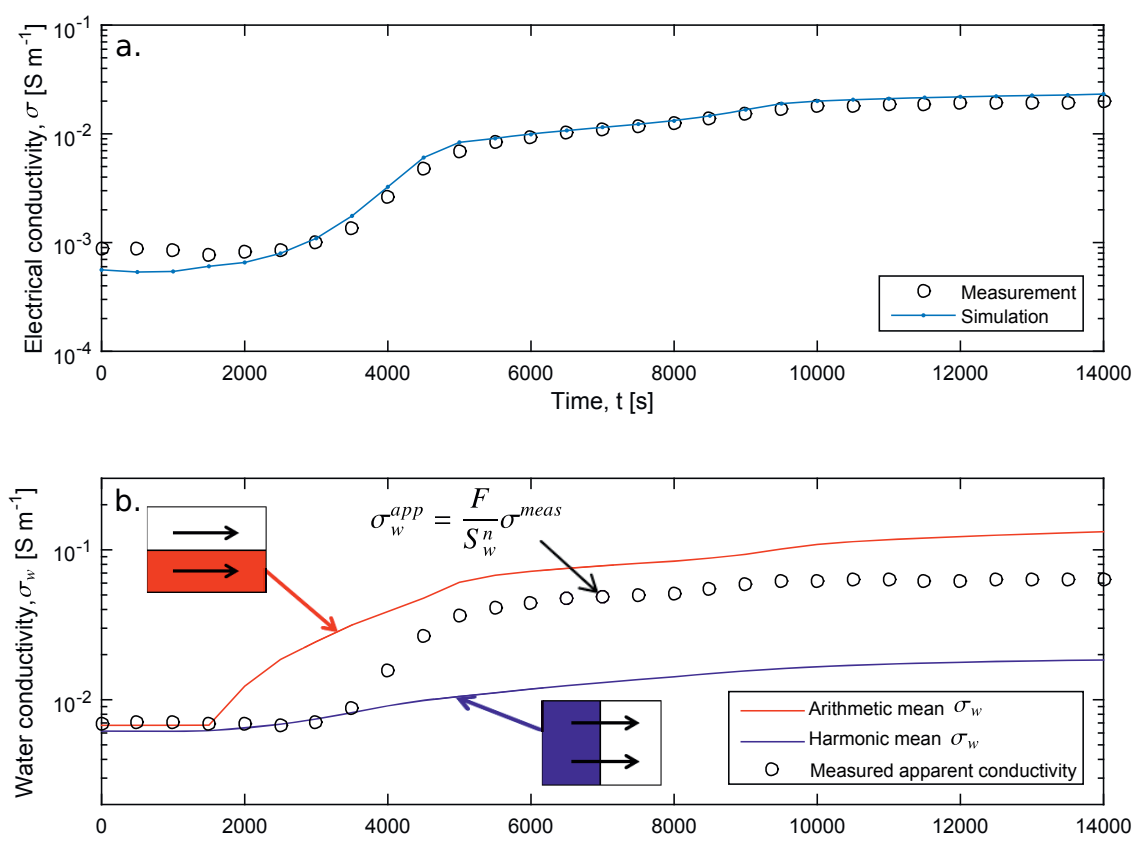

Figure 8: (a) Comparison between measured and simulated electrical conductivities at the scale of the flow cell under partially saturated conditions $\left(R M S E=0.0017 \mathrm{~S} \mathrm{~m}^{-1}\right)$. (b) Apparent water electrical conductivity from Eq. 2 (with $F=1.85$ and $n=4$ ) and Wiener bound values of the wetting phase at the flow cell's scale. Note that the water saturation increased from $S_{\mathrm{w}}=0.69$ to 0.87 during the test. 


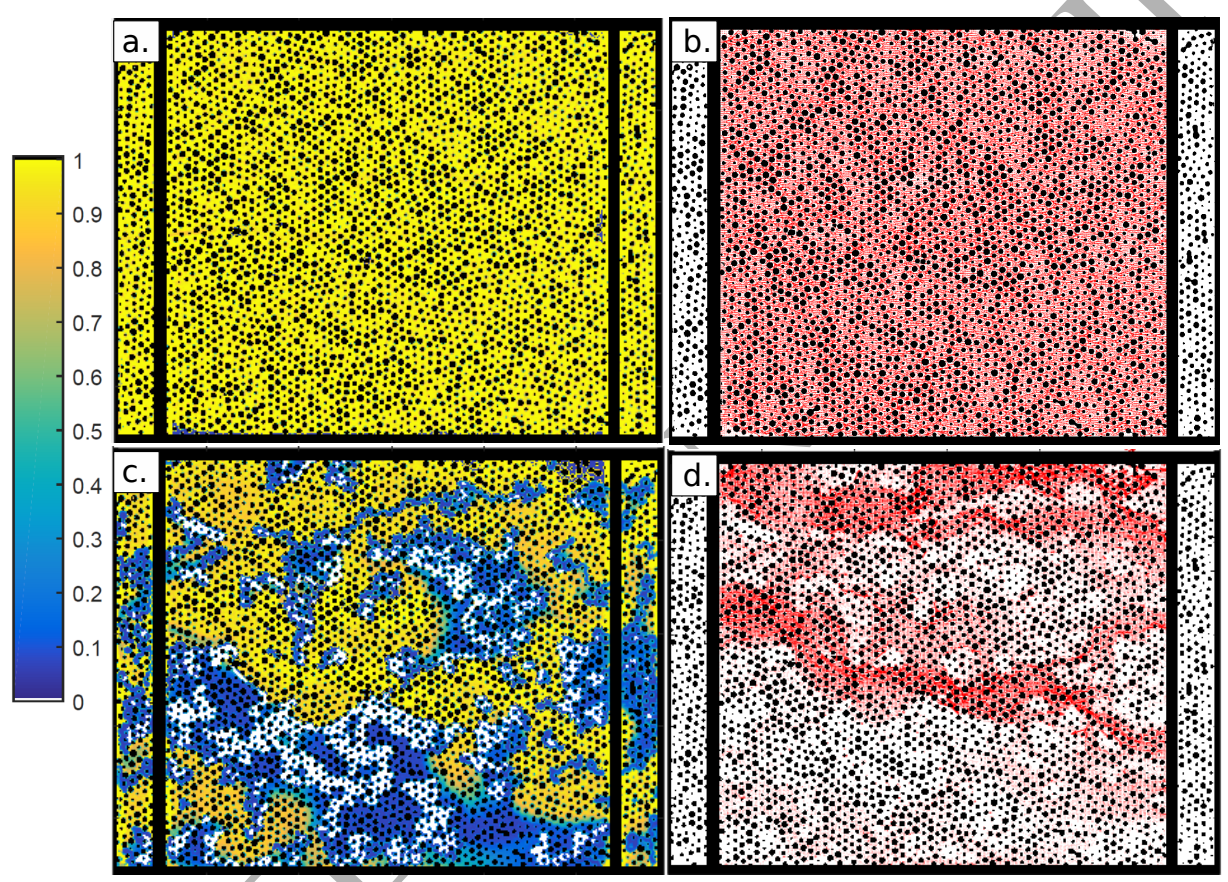

Figure 9: ( $a$ and c) Normalized tracer concentration and (b and d) current density spatial distribution at a late stage of the tracer test experiments under saturated ( $a$ and $b$ correspond to $t=12000 \mathrm{~s}$ ) and partially saturated (c and d correspond to $t=14000 \mathrm{~s}$ ) conditions. Note that we only display the current density as red streamline density between P1 and P2, masking the effect of the high conductivity of the copper electrode to improve the readability of Figs. $9 \mathrm{~b}$ and $\mathrm{d}$. 
a.
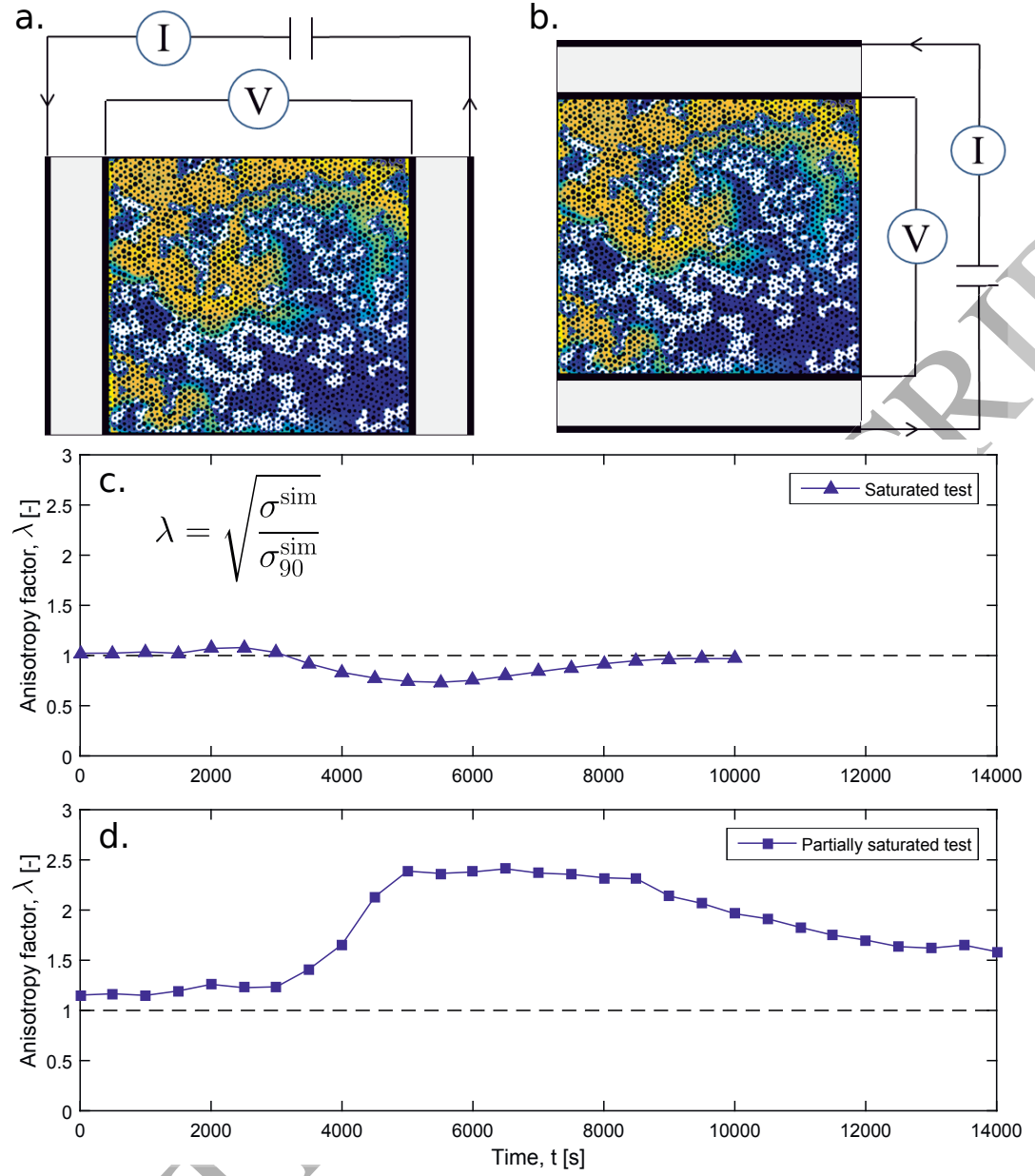

e.

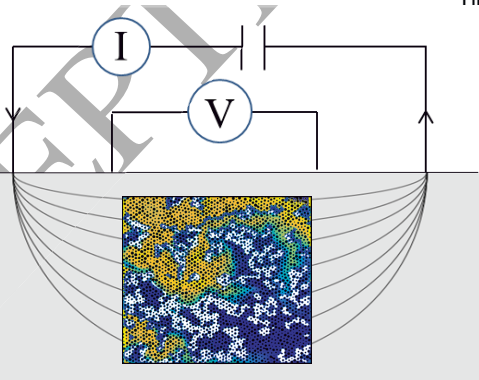

b.

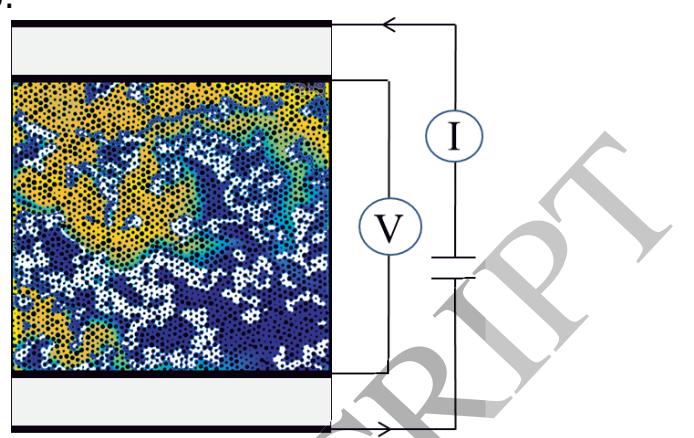

f.

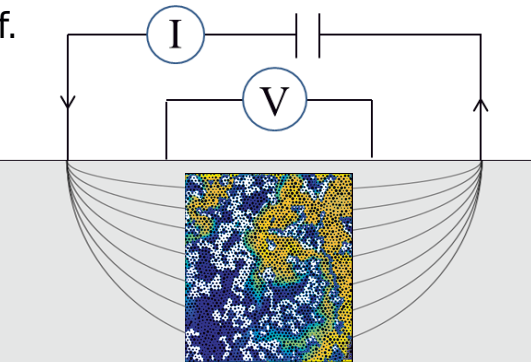

Figure 10: Numerical study of anisotropy in the effective bulk electrical conductivity during tracer experiments for (a) the experimental configuration with longitudinal electrical transport and (b) a rotation of the set of electrodes with respect to the medium by $90^{\circ}$ (i.e., imposing transverse electrical transport with respect to the main flow direction). (c) The electrical anisotropy factor as a function of time during the saturated tracer test. (d) Same as (c) for partially saturated conditions. Note that the anistropy study is only conducted on the part of the cell between P1 and P2 (i.e., the investigation zone). The dashed line corresponds to the value $\lambda=1$. Conceptual view of surface-based ERT measurements for (e) lateral and (f) vertical tracer flow in the near surface. 